\title{
MedienPädagogik
}

Zeitschrift für Theorie und Praxis der Medienbildung

Jahrbuch Medienpädagogik 4.

Zweitveröffentlichung aus: Jahrbuch Medienpädagogik 4. (2005) Wiesbaden: VS Verlag für Sozialwissenschaften. Hrsg. v. Ben Bachmair, Peter Diepold und Claudia de Witt.

\section{Navigationsverhalten im Internet \\ Eine qualitative Analyse von typischen Suchstrategien der Nutzer des Deut- schen Bildungsservers}

\author{
Michael Kluck und Susanne Politt
}

Aufgabe von Bildungsportalen ist es, den „Normalnutzern“ den Zugang zu den gewünschten Informationen zu erleichtern. Die bisher vorliegenden empirischen Untersuchungen über das reale Suchverhalten von Internetnutzern beziehen sich im wesentlichen auf allgemeine Suchmaschinen. Ziel der vorliegenden Studie ist es, das Suchverhalten und die typischen Suchanfragen von Normalnutzern von Bildungsportalen in einer qualitativen Analyse zu untersuchen. Grundlage dafür waren Logfiles des Deutschen Bildungsservers aus dem Zeitraum Oktober 2001 bis April 2002. Diese wurden bearbeitet und zum einen auf typische Suchanfragen hin untersucht, zum anderen wurden Erkenntnisse über individuelle Suchstrategien herausgearbeitet und qualitativ analysiert. Diese Ergebnisse werden mit den Resultaten anderer empirischer Untersuchungen verglichen. Einige Schlussfolgerungen für die Weiterentwicklung von Bildungsportalen bilden den Abschluss der Untersuchung.

\section{Fragestellung der Untersuchung}

Diese Studie beruht auf unseren Arbeiten zum Vergleich der Leistungsfähigkeit von Suchmaschinen in dem Gegenstandsbereich Bildung, Erziehung, Schule. Im Zuge dieser Untersuchungen haben wir auch die Aufzeichnungen und Auszählungen der Logfiles bezüglich der Suchanfragen an den Deutschen Bildungsserver ausgewertet. Die speziellen Einsichten in das reale Suchverhalten beim Deutschen Bildungsserver schien es wert zu sein, gesondert dargestellt zu werden.

Ausgehend vom aktuellen Forschungsstand soll daher in dieser qualitativen Studie das reale Nutzerverhalten bei Anfragen an Bildungsservern untersucht werden und zwar anhand von Daten des „Deutschen Bildungsservers“. Die Fragestellung der Studie geht in zwei Richtungen: Zum einen wird untersucht, welche „typischen Fragenstellungen“ Nutzer von Bildungsservern - in diesem Fall des Deutscher Bildungsservers - haben, d.h. welche Themen und Bereiche vor allem gesucht bzw. nachgefragt werden. Zum anderen werden individuelle Suchstrategien nachverfolgt und vorsichtige Aussagen über typisches Suchverhalten gemacht. 


\section{Bisherige Untersuchungen zum Suchverhalten von Internetnutzern, Stand der Forschung}

Zum tatsächlichen Suchverhalten von Endnutzer liegen nicht viele quantitative empirische Untersuchungen und auch nur wenige qualitative Untersuchungen vor, dies gilt erst recht für die Nutzer von Bildungsportalen bzw. die Nutzergruppen Schüler oder Lehrer (siehe auch Feil 2000). Die meisten empirischen Untersuchungen mit Benutzern finden mit Studenten der Informatik oder Informationswissenschaft statt, um Systemleistungen und Benutzerverhalten zu testen. Außerdem gibt es einige generelle Aussagen über Internetnutzer, die auf allgemeinen Statistiken der Suchmaschinenanbieter beruhen, aber nicht das Vorgehen der einzelnen Benutzer betreffen, sondern nur Überblicksinformationen zu Suchwörtern, Seitenabrufen, Iterationen der Suche usw. bieten.

Die wesentliche Aussage dieser allgemeinen Betrachtungen ist, dass die Benutzer zu mehr als 95\% nur ein bis zwei Suchbegriffe eingeben, maximal zwei Seiten Ergebnisse anschauen und schnell neue Suchen beginnen, ohne die erste Suche tatsächlich für die Verfeinerung ihrer ursprünglichen Anfrage zu nutzen. Die meisten Benutzer sind sich offensichtlich auch über die Funktionalitäten der jeweiligen Suchmaschinen nicht klar und können weder Boolesche Logik noch Trunkierung oder andere Zusatzfunktionen richtig verwenden oder einschätzen. Schließlich sind sie nicht in der Lage, die Ergebnispräsentation zu verstehen, die meist auf statistisch ermitteltem Ranking beruht, was auch durch die Geheimhaltung dieser Mechanismen seitens der Suchmaschinenanbieter erschwert wird.

In generellen Untersuchungen zum Verhalten von Internetnutzer wurde hinsichtlich Alta Vista und Excite folgendes Benutzerverhalten beschrieben (Silverstein et al. 1998, Spink/Xu 2000, Wolfram et al. 2001):

- Bei 42\%- bzw. 85\% der Suchanfragen wird nur der erste Bildschirm mit Resultaten betrachtet.

- Ca. zwei Drittel der Sitzungen umfassen nur 1 Frage (ohne weitere Modifikation der Anfrage im weiteren Verlauf).der Anfragen enthalten mehr als 3 Suchbegriffe.

- Von den jeweiligen Benutzern werden 1-2 Anfragen pro Sitzung gestellt.

- Nur 1-2 Seiten der Ergebnisliste werden angesehen; 70\% der Benutzer sehen sich nur die ersten zehn Treffer an.

- Boolesche Operatoren (einschließlich „,“) werden bei 5\% bis zu einem Drittel der Anfragen eingesetzt (damit ist aber noch nicht gesagt, dass sie richtig verwendet werden: mindestens jeder zweite Benutzer macht dabei Fehler).

- Die Suche von Phrasen (Begriffe, die in Anführungszeichen gesetzt sind) wird nur von ca. $6 \%$ genutzt.

- In ca. 5-10 \% der Anfragen wird bei Excite das Relevanzfeedback genutzt. 
- Ca. 5\% der Anfragen betreffen den Bereich Bildung und Geisteswissenschaften.

Für Web-Neulinge oder Gelegenheitsbenutzer wurde zum Beispiel festgestellt (Hölscher/Strube 2000), dass sie

- die angebotenen einfachen und komplexen Operatoren und Mechanismen kaum nutzen, mit Ausnahme des „+-Operators“.

- seltener direkt eine bestimmte Suchmaschine ansprechen, sondern eher auf den Suchknopf von Netscape Navigator/Internet Explorer drücken, um eine Suche zu starten.

- öfter die Suchanfrage wiederholen oder wieder neu beginnen, statt die ersten Ergebnisse näher zu betrachten und die Suchanfrage weiter zu verfeinern.

- öfter zu vorherigen Ergebnissen/Schritten zurückkehren als erfahrene Benutzer.

- wesentlicher häufiger Fehleingaben machen (Schreib- und Strukturfehler).

- generell größere Probleme mit der Formulierung sinnvoller Anfragen haben.

Für Kinder und Schüler wurden folgende Verhaltensweisen bei der Internetsuche ermittelt (Schulz 1998: zusammenfassende Übersicht über einige Untersuchungen; Schacter et al.1998; Feil 2000: zusammenfassende Studie des Deutschen Jugendinstituts; Large/ Behesti 2000; Neumann 1995; Priemer/Schön 2002a, 2002b: Studie mit Schülern im Fach Physik der Sekundarstufe) (- hier nur die Angaben zu mehr als 10 Jahre alten Kindern und Schülern):

- Frage- und Suchformulierung stellen ein großes Problem dar. Primarschüler haben Probleme, effektive Suchbegriffe auszuwählen. Sie wissen nicht, welche Begriffe sie für die Suche wählen sollen.

- Schüler sind eher passive Empfänger von Aufgaben, unkritisch gegenüber dem Wert und der Angemessenheit der gefundenen Information aus dem Internet. Kinder schätzen alle gefundenen Informationen als in hohem Maße glaubwürdig ein.

- Schüler arbeiten eher unsystematisch, ohne Überprüfung der eigenen Vorgehensweise. Kinder wenden bei der Suche im Internet reaktive Strategien an, d.h. sie hangeln sich von einem gefundenen Ergebnis zum anderen und planen ihre Internetsuche nicht systematisch. Schüler neigen ebenfalls eher zu blinder Aktivität und arbeiten eher interaktiv als systematisch.

- Schüler haben Schwierigkeiten mit dem Alphabet und mangelhaftes Lesevermögen.

- Meistens browsen die Kinder durch die Datenbestände und orientieren sich nicht an analytischen, schlagwort-gestützten Suchstrategien. Offene Fragestellungen können sie deshalb eher mit Hilfe des Internet beantworten als exakt definierte Aufgaben. 
- Lehrer überschätzen die Informationskompetenz der Schüler.

- Die Relevanzbewertung fällt Kindern schwer.

- Hilfeinformationen werden von Kindern vollständig ignoriert, sie konzentrieren sich auf die Bildschirmmitte.

- Erst ab 10/11 Jahren beginnen Kinder abstrakt zu denken und entsprechende Strukturen (z.B. Klassifikationen, Verweise) zu nutzen.

- Nach einem Null-Ergebnis sind viele Kinder ratlos und finden keine alternativen Formulierungen.

- Von Kindern werden überwiegend einfache, konkrete Suchbegriffe eingegeben, komplexe Suchformulierungen bilden ein Problem und sind häufig Fehlerquellen. Primarschüler haben um so mehr Probleme je mehr Konzepte in eine Suchstrategie eingebaut werden müssen. Primarschüler sind erfolgreicher, wenn sie mit konkreten Aufgaben befasst sind als bei abstrakten Problemen. Kinder sind nicht so gut in der Lage, mit der Sprache zu spielen und Suchstrategien zu formulieren. Kinder haben sogar bei speziell für Kinder konstruierten Suchmaschinen Probleme mit der Suchformulierung. Kinder und Jugendliche sind oft nicht in der Lage, mit Suchmaschinen im Internet umzugehen, und können viele Angebote noch nicht ohne Unterstützung oder Unterweisung durch Erwachsene nutzen.

- Logische Operatoren und andere Funktionalitäten werden von Kindern nicht verstanden und ggf. widersinnig verwendet (wie bei Erwachsenen!). Kindern fehlt das für eine erfolgreiche Suche im Internet wichtige Verständnis, wie Suchmaschinen aufgebaut sind und funktionieren.

- Domänenspezifische Vorkenntnisse sind hilfreich für das Lernen mit dem Internet.

- Die Fähigkeit, kritisch mit den Informationen umzugehen, ist ausschlaggebend für den Lernerfolg mit dem Internet.

- Gezieltes Suchen und globales Überblick-Verschaffen sind sehr erfolgreiche Strategien.

- Schüler, die viel Text aus dem Internet kopieren, haben schlechtere Lernerfolge als solche, die die Texte weitgehend selbst verfassen.

- Schlüsselqualifikation ist die Fähigkeit zur Selektion der Quellen.

Das Interesse der Lehrer wiederum „konzentriert sich auf Material, das möglichste direkt oder mit geringem Bearbeitungsaufwand im Unterricht genutzt werden kann. (...) Diese Art von ,Einsetzbarkeit' des ,Materials’ wird zu einem zentralen Kriterium der Bewertung des Ertrags der Internetnutzung zur Informationsrecherche. Damit wird die Informationsrecherche auf eine Unterrichtsmaterialsuche verengt“ (Hedtke/ Kahlert/ Schwier 1998, S. 366f).

Für Hochschulstudenten wurde in einer gerade veröffentlichten Untersuchung festgestellt, dass sie wesentlich mehr Wert auf einfache (= leichte) Benutzbarkeit legen als auf das Retrieval genauer Ergebnisse: „Participants consistently desired an easy and familiar search process - regardless of the search’s results“ (Augustine/Greene 2002). 
Generell kann man davon ausgehen, dass sich die Ergebnisse hinsichtlich des Suchverhaltens (nicht aber in bezug auf die Interpretation der Nützlichkeit) für Kinder über 10 Jahren und Schüler weitgehend auch auf Erwachsene, d.h. auch Lehrer übertragen lassen. Denn die Befunde für Nutzer im allgemeinen stimmen weitgehend mit denjenigen zu den spezielleren Nutzergruppen Schüler und Kinder über 10 überein.

Die Untersuchung der Nutzerstruktur des Deutschen Bildungsservers hat ergeben, dass die große Mehrheit der Benutzer Lehrer und Lehrende im weitesten Sinne (64\%) sind, die Schüler, Studenten und Auszubildenden (15\%) spielen eine deutliche geringere, aber dennoch nicht zu vernachlässigende Rolle ${ }^{1}$ in der Nutzerschaft. Daher ist zu erwarten, dass die vorliegenden Befunde durch die Analyse der konkreten Suchanfragen weitgehend bestätigt werden.

\section{Datengrundlage der Untersuchung, Aufbereitung der Daten und Vorgehen bei der Analyse}

\section{Datengrundlage ${ }^{2}$}

Als Grundlage dienten Logfiles (= Mitschnitte der Nutzeraktionen) des Deutschen Bildungsservers aus dem Zeitraum Oktober 2001 bis 17. April 2002 in folgenden Fassungen:

a) in vollständiger Fassung (Suchworte - Verknüpfungsart - anfragender Rechner - Datum/Zeit)

- nach Monaten aufgesplittet

- nach anfragenden Servern und nachfolgend chronologisch sortiert.

Diese Dateien sind nutzbar für Erkenntnisse über individuelles Suchverhalten.

b) nur die Anfragen selbst, nach Häufigkeit sortiert

Die jeweiligen Anfragen Suchanfragen als Phrase oder String (= Zeichenkette des gesamten eingegebenen Textes) hintereinanderweg, ggf. mehrere Wörter oder Wortgruppen.

Diese Dateien sind so nicht mehr nutzbar für Erkenntnisse über individuelles Suchverhalten, liefern aber Anhaltspunkte für den Inhalt der Anfragen und deren Formulierung.

1 Vgl. den Vortrag von Brenstein/Kos auf dieser Tagung, dort auch näheres zu den Nutzerdaten.

2 Die Daten der Logfiles wurden freundlicherweise von Dr. Christian Richter vom Deutschen Bildungsserver zur Verfügung gestellt, technisch handhabbar gemacht und aufbereitet. 


\section{Vorgehen zur Untersuchung der häufigsten Fragen}

Als Grundlage für die Untersuchung der häufigsten Fragen dienten die nach Häufigkeit sortierten Abfragen der Wortgruppen. Diese Datei wurde nach folgenden Kriterien bearbeitet:

Alle Begriffe, die nur ein- bis dreimal abgefragt wurden, sind jeweils in gesonderte Dateien aussortiert worden. In diesen drei Dateien wurde der absolute Unsinn gelöscht (bzw. ggf. nachgebessert wie unten in der „Hauptdatei“). Diese Dateien enthalten oft Spezialfälle der häufigeren Anfragen bzw. interessante Kombinationsabfragen, sie können für die Formulierung typischer Suchanfragen genutzt werden. Diese typischen Suchanfragen werden auch für Tests in einem anderen Projekt (Web Search Bench ${ }^{3}$ ) verwendet.

In der so entstandenen Datei wurden alle Begriffe, die häufiger als 20mal vorkamen, als „Oberbegriffe“ herausgezogen (halbfett markiert). Dann wurde alphabetisch sortiert, in der Ergebnisdatei wurden Tippfehler korrigiert und inhaltlich ähnliche bzw. gleiche Abfragen zusammengefasst, Singular und Plural des gleichen Oberbegriffs wurden zusammengefasst. Das heißt, dass man diese Datei nicht mehr für Erkenntnisse zum Suchverhalten (auch nicht zum allgemeinen!) nutzen kann, weil die Begriffe umformuliert, nachbearbeitet und zusammengefasst wurden. Aus dieser Datei wurden jedoch die typischen Fragebereiche herauskristallisiert, da die Oberbegriffe eine Kategorisierung der Suchanfragen nahe legten.

\section{Vorgehen zur Untersuchung individueller Suchstrategien und typischen Such- verhaltens}

Um individuelle Suchstrategien zu erkennen, wurden die zeitlich aufeinanderfolgenden Anfragen, die von einem Rechner kamen, isoliert. Diese (zeitlich) zusammenhängenden Fragefolgen wurden markiert und untersucht. Dies geschah exemplarisch am Beispiel derjenigen Rechner, die vier und mehr Anfragen in Folge durchgeführt hatten. Auf dieser Basis lassen sich eher Strukturen erkennen, außerdem ist für eine vorläufige Untersuchung die Datenmenge in ihrer Gesamtgröße sonst nicht zu handhaben. Die zweifachen und dreifachen Anfragen wurden jedoch zur Kontrolle der gebildeten Gruppen des Anfrageverhaltens hinzugezogen.

Die ODER-Anfragen (Typ: gesucht sind Ergebnisse, die die Begriffe A oder B enthalten) sind in den Beispielen rot markiert, kursiv gesetzt und un-

3 Web Search Bench (ursprünglich: Intelligente Internet-Suchmaschine) ist ein vom DFN gefördertes Projekt in dem die Humboldt-Universität als fachspezifische Benutzergruppe mit den Daten des Deutschen Bildungsservers beteiligt ist. Siehe http://www4.cs.uni-dortmund.de/ Lindemann/projects/SearchEngine.html. 
terstrichen, alle anderen Anfragen sind UND-Anfragen (Typ: gesucht sind Ergebnisse, die gleichzeitig die Begriffe A und B enthalten).

Die einfachen Anfragen (von einem spezifischen Rechner aus wurde nur ein Suchwort eingegeben, dann wurde die Suche beendet) wurden in einer gesonderten Datei gesammelt. Anmerkungen dazu folgen weiter unten.

Einschränkende Vorbemerkungen: Die Datengrundlage ist zum einen zeitlich begrenzt, zum anderen beruht sie ausschließlich auf einem Beispiel, nämlich dem Deutschen Bildungsserver, daher sind die Ergebnisse cum grano salis zu betrachten. Weitere Einschränkungen ergeben sich durch Aufbereitung der Daten. Bei den Schlussfolgerungen handelt es sich daher um vorläufige Ergebnisse, die zu diskutieren und weiterhin zu überprüfen wären.

\section{Ergebnisse der qualitativen Analyse}

Typische Fragen von ,Normalnutzer' von Bildungsportalen

Auf der Grundlage der oben genannten Logfiles wurde die Gruppierung und die Nennungen von einzelnen Beispielen vorgenommen, die keineswegs abschließend oder vollständig sind. Die Zahlen in Klammern beruhen auf einer einfachen Auszählung, die lediglich die Größenordnung der Nennungen andeuten kann. Die Auszählungen enthalten nur die Oberbegriffe mit Varianten (Singular/Plural, Schreibfehler, Komposita) ohne weitere Spezialbegriffe, die ebenfalls einschlägig wären4

\section{Unterrichtsfächer und Unterrichtsinhalte}

alle Unterrichtsfächer: Mathematik bzw. „Mathe“ (852+236), Biologie (653), Deutsch (621), Geschichte (537), Chemie, Physik, Englisch, Musik, Informatik, Erdkunde, Latein, Religion, Sport, Französisch, Kunst, DAF bzw. Deutsch als Fremdsprache (alle $>200$ ).

spezielle Unterrichtsinhalte: Euro (335), Wasser (379), Internet (309), Märchen (224), E-Learning (303), Referate (401), Bewerbung (329), Mittelalter (190), analytische Geometrie (169), Elektrolyse (25), Balladen (149), Erörterung (310), Interpretation (343), Französische Revolution (111), Pythagoras (99).

Schriftsteller (ohne einzelne Werke): Goethe, Dürrenmatt, Brecht, Schiller (alle > 87)

Gezielte Literaturanfragen: Faust (167), „als Hitler das rosa Kaninchen stahl“, z.B. „Bahnwärter Thiel“, “Nathan der Weise“ (87), „Hauen ist doof“.

\section{Lehrpläne, Rahmenrichtlinien, Stoffverteilungspläne}

Lehrplan bzw. Lehrpläne jeweils für bestimmte Fächer bzw. in bestimmten Bundesländern.

4 Eine eher klassifikatorische Auszählung der hier verwendeten Daten ist bei Brenstein/Kos zu finden. 
III. „Pädagogisches“

(Fach-)Didaktik, Freiarbeit, fächerübergreifender Unterricht, differenzierter Unterricht, Projekte, Handlungsorientierung, Gruppenarbeit, Hochbegabung, Wochenplan, Facharbeit.

\section{Prüfungen und Bewertungen}

Klausuren, Abiturfragen (für spezifische Fächer), Tests, Klassenarbeiten, Examen, Prüfung; Bewertungskriterien für... , Benotung, Beurteilungskriterien.

\section{Zeittypische bzw. aktuell diskutierte Themen}

Ferien (452), Weihnachten (159), PISA-Studie (alle Varianten addiert: 2576), Karneval (29), Frühling (58), Ostern, Abitur (> 1000 ohne fachspezifische Abituraufgaben).

\section{Lehrer...}

Lehrerausbildung, Lehrerberuf, Lehrerfortbildung (68), Lehrerrolle.

\section{Gesetzliches und Institutionelles, Verordnungen}

Schulgesetz von ... , z.B. Aufsichtspflicht, Fachhochschulreife in Bayern, Duales System.

\section{Geographisches}

einzelne Bundesländer, Städte, Regionen, Länder...

\section{Berufe}

Altenpflegerin, Arzthelferin, Bankkaufmann, Bilanzbuchhalterin.

\section{$X$. Institutionen, bestimmte Schulformen}

Grundschule, Berufsakademie, gymnasiale Oberstufe (sic!, also nicht eine ganz bestimmte Schule irgendwo, sondern die Schulform).

\section{Typisches Suchverhalten, individuelle Suchstrategien: Erkenntnisse und Ver- mutungen}

Grundlage hierfür waren die Dateien mit den individuellen Abfragen, d.h. mit den zeitlich aufeinander folgenden Anfragen eines Rechners. Die Ergebnisse werden hier jeweils mit einigen Beispielen dargestellt, weitere Beispiele befinden sich in gesonderten Dateien. ${ }^{5}$

Erwartetes und bestätigtes, vernünftiges 'Verhalten

Wie erwartet ließen sich die typischen Suchstrategien des Einschränkens bzw. des Ausweiten finden, ebenso die Versuche, Begriffe zu umschreiben bzw. ,einzukreisen` (mit der Unterform, verschiedene Schreibweisen auszuprobieren).

5 http://www.f4.fhtw-berlin.de/ politt/suchverha1ten_beispiele.doc 
Ebenfalls häufig anzutreffen waren Versuche der ,Trunkierung', wobei meist auf gängige Formen zurückgegriffen wird, (offenkundig ohne dass die spezifischen Hilfe-Seiten des Deutschen Bildungsservers gelesen wurden). Generell wird versucht, Erfahrungen aus Recherchen mit anderen Suchmaschinen zu übertragen.

Bsp. Einschränken:

\begin{tabular}{lll}
\hline PENTOMINO & Anfrage A & 20011031153850 \\
PENTOMINO UND HEXOMINO & Anfrage A & 20011031153957 \\
\hline & & \\
Bsp. Ausweiten: & & \\
\hline neuer Lehrplan & Anfrage B & 20011022114804 \\
Lehrplan & Anfrage B & 20011022114826 \\
\hline
\end{tabular}

Bsp. Einkreisen:

\begin{tabular}{lll}
\hline FREIWILLIGE & Anfrage D & 20011024135800 \\
EHRENAMT & Anfrage D & 20011024135823 \\
\hline
\end{tabular}

Bsp Trunkierung bzw. Versuch der Übertragung bisheriger Erfahrungen:

\begin{tabular}{lll}
\hline GEWALTPRAEVENTION & Anfrage I & 20011212181544 \\
GEWALT.\% SCHULE.\% & Anfrage I & 20020128161554 \\
GEWALT.\% SCHULE.\% & Anfrage I & 20020128161623 \\
GEWALT GRUNDSCHULE & Anfrage I & 20020128161714 \\
\hline & & \\
\hline ENTWICKLUNG DES MAIKAEFERS & Anfrage K & 20020220205856 \\
ENTWICKLUNG UND DES UND MAIKAEFERS & Anfrage K & 20020220210100 \\
ENTWICKLUNG DES MAIKAEFERS & Anfrage K & 20020220210648 \\
DIE ENTWICKLUNG DES MAIKAEFERS & Anfrage K & 20020220210945 \\
DIE_ENTWICKLUNG_DES_MAIKAEFERS & Anfrage K & 20020220211049 \\
DIE UND ENTWICKLUNG UND DES UND MAI- & Anfrage K & 20020220211126 \\
KAEFERS & & \\
\hline
\end{tabular}

\section{Probleme / unerwartetes Verhalten}

\section{Tippfehler}

Wenn die Nutzer ihre eigenen Tippfehler bemerken und nachbessern, ist es nicht so problematisch. Schwierig wird es, wenn sie die Tippfehler nicht bemerken und dann stattdessen bemüht sind, die Suchbegriffe umzuformulieren, obwohl diese ganz vernünftig waren. 


\begin{tabular}{lll}
\hline BETRATUNGSLEHRER & Anfrage L & 20011025122001 \\
SCHULBERATUNG & Anfrage L & 20011025122021 \\
\hline
\end{tabular}

\section{Wiederholung}

Manche Nutzer geben dieselbe Abfrage mehrmals hintereinander ein. Bei der Interpretation sollte man vorsichtig sein, weil das sehr unterschiedliche Ursachen haben kann:

Meistens handelt es sich wahrscheinlich einfach um eine Neueingabe, jedes Mal nachdem man ein einzelnes Beispiel genauer angesehen hat ${ }^{6}$. Manchmal wird aber auch dieselbe Abfrage mehrmals hintereinander eingegeben, obwohl kein Ergebnis bzw. nur ein Ergebnis erzielt wurde. (Es ist nicht ganz klar warum. Die Nutzer meinen vielleicht, es hat nicht geklappt bzw. wollen vorsichtshalber mehrmals „auf den Knopf drücken“?)

\section{Beispiel:}

Ein Nutzer hat am 20.10.2001 über einen Zeitraum von mehr als 60 Minuten 18mal das Wort TALIBAN eingegeben, in unterschiedlicher Schreibweise, mal als UND- und mal als ODER-Verknüpfung. Der Grund ist unklar. (In diesem Fall war der Grund NICHT der, dass verschiedene Ergebnisse angesehen wurden! Es gab nur eins.)

\section{„alle Wörter" in getrennten Abfragen}

Manche Nutzer scheinen eine UND-Suche mit verschiedenen Begriffen anzustreben, indem sie jedes Wort einzeln eingeben, die Variante „alle Wörter“ wählen und dann jedes Mal für das einzelne Wort eine gesonderte Suche starten. Eventuell erwarteten sie, dass die Einzelabfragen gespeichert und kombiniert werden.

\section{ODER-Verknüpfungen}

Oder-Verknüpfungen werden sehr selten genutzt, auch wenn es eigentlich sinnvoll wäre; i.a. werden die vorgefundenen Voreinstellungen übernommen.

Negativbeispiel

\begin{tabular}{|c|c|c|c|}
\hline ERWACHSENENPAEDAGOGIK WEITERBIL- & UND & Anfrage Z & 20011030202924 \\
\hline
\end{tabular}

\section{UND-Verknüpfungen}

UND-Verknüpfungen sind zwar häufiger als ODER-Verknüpfungen, die Mehrheit der Benutzer benutzt aber generell kaum die Möglichkeit,

6 Bei speziellen Browser-Voreinstellungen (Cache=0) kann es auch sein, dass die Seite jedesmal neu „geholt“ wird und deshalb jedesmal als neue Anfrage in den Logfiles auftaucht. Das ist allerdings eher unüblich. 
mehrere Wörter zu kombinieren. Am häufigsten werden Einwort-Abfragen gestellt.

unsystematisches Vorgehen

Die Behauptung, dass ein Großteil der Nutzer sehr unsystematisch vorgeht, wird durch unsere Untersuchung bestätigt.

Ein Extrembeispiel für eine sehr ausdauernde unsystematische Suche:

Die folgenden Begriffe wurden über einen Zeitraum von über 15 Minuten ohne Verknüpfung nacheinander, z.T. auch mit Wiederholung bereits durchgeführter Abfragen eingegeben:

GESCHICHTE, KREUZZUEGLER, GESCHICHTE, KREUZZUEGE, GESCHICHTE, MITTELALTER, MITTELALTER, MITTELALTER, LEUTE DES MITTELALTERS, KREUZRITTER, MITTELALTER, MITTELALTER, GESCICHTE, GESCICHTE, MITTELALTER, MITTELALTER, GESCICHTE, PABST URBAN 2, MITTELALTER, PABST URBAN 2, MITTELALTER, MITTELALTER, MITTELALTER, MITTELALTER,

Lange Texte

Häufig sind umgangssprachliche Eingaben von längeren Phrasen mit allen Verbindungsund Füllwörtern.

\begin{tabular}{l}
\hline WELCHE AEMTER SIND DEN HAUPTSCHULEN \\
UEBERGEORDNET?
\end{tabular}

„Mal gucken“"

Häufig scheint es sich nicht um eine gezielte Suche zu handeln, sondern eher um ein interessiertes Herumgucken, was es denn so gibt:

Bespiel:

BIOLOGIE, GRUNDSCHULE, GRUNDSCHULE DEUTSCH, GRUNDSCHULE ARBEITSBLAETTER, ARBEITSBLAETTER BIOLOGIE, ARBEITSBLAETTER GESCHICHTE, ARBEITSBLLAETTER SPORT, ARBEIT \% SPORT, ARBEITSMATERIALIEN GRUNDSCHULE,

Kommunikation mit dem Rechner

Manchmal scheint es, als würde die Suchmaschine nicht nur als technisches Hilfsmittel genutzt, sondern als würden die Nutzer mit dem Rechner „kommunizieren“. Wenn eine Suche nicht klappt und die gewünschten Ergebnisse nicht erzielt werden, geben anscheinend manche Nutzer ihre Ratlosigkeit 
oder ihre Unmutsäußerung gleich noch mal als Abfrage ein. (Bsp.: WO HIN WENN MAN WAS SUCHT?; Bsp: fuck u).

\section{Anmerkungen zu den 1er-Anfragen}

Viele Nutzer starten nur eine Abfrage und beenden die Recherche dann gleich wieder. Die Motive hierfür (haben sie aufgegeben? haben sie sofort das Gewünschte gefunden und sind zufrieden?) lassen sich nur vermuten, sie sind mit dieser Studie nicht festzustellen.

\section{Schlussfolgerungen, Vermutungen, Hypothesen}

- Der überwiegende Teil der Nutzer sucht nur mit einem Begriff, ohne die Möglichkeit zu nutzen, mehrere Begriffe zu verknüpfen.

- ODER-Verknüpfungen werden fast gar nicht benutzt, weil wahrscheinlich einfach die vorgefundenen Standardeinstellungen übernommen werden, (in diesem Falle diejenigen des Deutschen Bildungsservers: logisches Und) und keine Kenntnis der anderen Möglichkeiten besteht, natürlich erst recht nicht hinsichtlich der Booleschen Logik.

- Die meisten Benutzer scheinen bestimmte Erfahrungswerte aus anderen Suchmaschinen zu übertragen, ohne nachzuprüfen, ob dies für die Suche beim Deutschen Bildungsserver auch zutrifft bzw. ohne die Hilfe-Seiten des Deutschen Bildungsservers zu nutzen: z.B. Trunkierung mit „*“ oder „\%“, z.B. eine Phrasensuche in Hochkommata, also 'neue Rechtschreibung'.

- Die meisten Nutzer erwarten anscheinend grundsätzlich eine trunkierte Suche, auch wenn sie kein „*“ oder „\%“ eingeben.

- Wenn mehrere Begriffe benutzt werden, geht es häufig um „String“-Abfragen von Phrasen, also z.B. den Titel eines literarischen Werkes, den Namen einer Institution, die Bezeichnung eines Faches oder eines didaktischen Vorgehens.

- Eine typische Anfrageform ist auch, dass ein Artikel mit eingegeben wird: z.B. „das Auge“, „der Igel“, „die Zelle“. Dies ist in den Auswirkungen aber im Allgemeinen unproblematisch, da die Artikel und andere Stoppwörter (= nicht-sinntragenden Begriffe) herausgefiltert werden.

Insgesamt ist eine große Übereinstimmung zu den allgemeinen Befunden zum Verhalten von Internetnutzern festzustellen, vielleicht mit der Ausnahme, dass tatsächlich in stärkerem Maße Fachvokabular als Suchterme verwendet wird. 
10. Thesen zu den Anforderungen an eine Suchmaschine für Bildungsportale

Für die Strukturierung von Internet-Suchbildschirmen gilt nach Shneiderman (1998, S. 511f).

„First-time users of an information-exploration system (whether they have little or much task knowledge) are struggling to understand what they see on the display while keeping in mind their information needs. They would be distracted if they had to learn complex query languages or elaborate shape-coding rules. They need low cognitive burdens of menu and directmanipulation designs and simple visual-coding rules. As users gain experience they can request additional features by adjusting control panels".

Am besten wäre also eine Suchmaschine, beider man gar nichts falsch machen kann. Die Grundregel für die Gestaltung ist daher: „Keep it simple!“ (Shneiderman 1998, Robertson 2001):

Für den Benutzer muss klar werden, was seine Eingabe bewirkt. Zunächst muss deutlich werden, welche Schreibweise im Eingabefeld zu welchen Resultaten führt. Dies sollte für die wesentlichen Anwendungsfälle direkt unter dem Eingabefeld erläutert werden, zusätzlich sollte ein Link auf „Tipps“ (nicht „Hilfe“!) verweisen, wo eine ausführlichere Beschreibung angeboten wird, die jedoch nach aller Erfahrung nur selten benutzt werden wird. Ferner sollte der Startkopf angemessen beschriftet sein: Text „Suche(n)“ und Icon „Lupe“. Die Standardsuche sollte ein logisches Und verwenden. Die Eingabe zweier oder mehrerer Stichworte sollte also weniger Treffer erbringen als vorher nur ein Stichwort.

Eine automatische Rechtstrunkierung wird nicht empfohlen. Denn hinsichtlich der Verwendung der Rechtstrunkierung als Voreinstellung ist Vorsicht geboten, da damit auch irrelevante Begriffe aus dem allgemeinen Wortschatz erfasst werden. Z.B. ergibt die Eingabe „schul“ bei Rechtstrunkierung neben Schule, Schulfach, Schulhof u.a. auch Schuld, Schulp, Schulter, Schultheiß u.a. Noch schwieriger wird es bei automatischer Links- und Rechtstrunkierung. Allenfalls könnte man sich vorstellen, Begriffe aus einem Fachthesaurus, wie dem ETB-Thesaurus ${ }^{7}$, als automatisch rechtstrunkiert zu verwenden ${ }^{8}$. Der Cursor sollte bereits im Eingabefeld für die Suchanfrage stehen.

7 ETB = European Schools Treasury Browser http://etb.eun.org ist ein europäisches Projekt zur Vernetzung von Bildungsportalen. Der ETB-Thesaurus ist ein in neun europäische Sprachen übersetzter Fachthesaurus für Online-Bildungsinformationen, an dessen Entwicklung die Autoren beteiligt waren.

8 Die oben genannte Suche „schul“ würde dann „nur“ folgende Thesaurus-Terme umfassen: Schulbibliothek, Schule, Schüler, Schüler-Schüler-Beziehung, Schüleraustausch, Schülerberatung, Schülerbriefwechsel, Schülerhilfe, Schülerzeitung, schulische Aktivitäten, schulische Autonomie, schulische Integration, Schulklasse, Schulleben, Schulleiter, Schulleitung, Schulmilieu, Schulpersonal, Schulpflicht, Schulversagen. Insgesamt wäre dies eine akzeptable Erweiterung, die den Sinn der Anfrage nicht wesentlich verfälscht, zumindest werden keine ganz abwegigen Terme in die Suche einbezogen. 
Die durchgeführte Aktion (Suche mit der Suchanfrage wie sie von der Suchmaschine abgearbeitet wurde) und die enthaltenen Suchbegriffe sollten auf der Ergebnisseite wiedergegeben werden: z.B. „die Suche nach „A und B“ ergab x Treffer“.

Im Hintergrund sollte die Suche durch eine fachspezifische Synonymliste unterstütz werden und eine Wortstammreduzierung (Stemming) stattfinden. Die Eingaben sollten durch ein Wörterbuch geprüft und gegebenenfalls korrigiert werden ${ }^{9}$ oder Schreibvarianten bzw. häufige Schreibfehler in die Synonymliste aufgenommen werden.

\section{Literatur}

Augustine, S./Green, C.: Discovering How Students Search a Library Web Site: A Usability Case Study. In: College and Research Libraries, 63 (4), 2002, pp. 354-365.

Brenstein, E./Kos, O.: Evaluation von Bildungsportalen - Empirische Untersuchungen zur Nutzung des Deutschen Bildungsservers und des Schulweb (Ergebnisse einer Nutzerprofilstudie), Beitrag in diesem Band, 2003.

Dickstein, R./Mills, V.: Usability Testing at the University of Arizona Library: How to Let the Users in on the Design. In: Information Technology and Libraries, Sept. 2000, pp. 144-151.

Feil, C.: Kinder im Internet. Angebote, Nutzung und medienpädagogische Perspektiven. In: DISKURS, 10, 2000, Heft 1, 15-24.

Hedtke, R./Kahlert, J./Schwier, V.: Unterrichtsmaterialien aus dem Internet: Eine empirische Studie über das Rechercheverhalten vom Lehrenden. In: Gegenwartskunde 47 (3), 1998, S. 363-375.

Hölscher, C./Strube, G.: Web Search Behavior of Internet Experts and Newbies. In: 9th International World Wide Web Conference, The Web: The Next generation, Amsterdam, May 15-19 2000, Conference Proceedings, available at: [http://www9.org/w9cdrom/81/81.html]

Jansen, B. J./Spink, A./Saracevic, T.: Real life, real users, and real needs: a study and analysis of user queries on the web. In: Information Processing and Management, 36 (2), 2000, pp. 207-227.

Large, A./Beheshti, J.: The Web as a Classroom Resource: Reactions from the User. In: Journal of the American Society for Information Science, 51 (12) 2000, pp. 1069-1080.

Naumann, A./Waniek, J./Krems, J. F.: Vergleich von Wissenserwerb und Navigationsstrategien bei linearen Texten und Hypertexten. In: W. Frindte, T. Köhler, P. Marquet, E. Nissen (Eds.). IN-TELE 99 - Internet-based teaching and learning 99. Frankfurt a. M. 2001, pp 305-310.

Neuman, D.: High School Students' Use of Databases: Results of a National Delphi Study. In: Journal of the American Society for Information Science, 46 (4), 1995, pp. 284-298.

Priemer, B./Schön, L.-H.: Physiklernen mit dem Internet. In: Deutsche Physikalische Gesellschaft (Hrsg.) Didaktik der Physik. Vorträge der Frühjahrstagung der DPG. Leipzig 2002, im Druck preprint unter [http://www.physik.hu-berlin.de/gruppen/didaktik/forschung/internet/priemer_ dpg_2002.PDF].

Priemer, B./Schön, L.-H.: Können Schüler Physik mit dem Internet lernen? In: Brechel, R. (Hrsg.): Zur Didaktik der Physik und Chemie. Berlin 2002, S. 186-188

Robertson, J.: Deploying an Effective Search Engine: Case study. 2001. [http://www.steptwo.com. au/papers/search/pdf/SearchEngine.pdf]

9 Eine Zwischenantwort wie z.B. bei Google vom Typ „Sie wollten wohl nach Y suchen“ scheint nur ein unnötiger, zeitraubender Umweg zu sein, wenn die korrigierten Suchterme zusammen mit dem Suchergebnis in der Beschreibung der durchgeführten Aktion genannt werden. 
Schacter, J./ Chung, G. K. W. K/ Dorr, A.: Children's Internet Searching on Complex Problems: Performance and Process Analyses. In: Journal of the American Society for Information Science, 49 (9), 1998, pp 840-849.

Schulz, U.: Das Projekt ,Kinder-OPAC‘ am Fachbereich Bibliothek und Information der FH Hamburg 1998. [http://www.bui.fh-hamburg.de/projekt/buecher-reise/kopart.htm].

Shneiderman, B.: Designing the User Interface: Strategies for Human-Computer Interaction. 3rd ed. Reading et. al: Addison-Wesley, 1998.

Silverstein, C./Henzinger, M./Marais, H./Moricz, M.: Analysis of a Very Large AltaVista Query Log. SRC Technical Note 1998-014.

Spink, A./Xu, J. L.: Selected results from a large study of Web searching: the Excite study. In: Information Research, 6 (1), 2000. [http://informationr.net/6-1/paper90a.html].

Wolfram, D./Spink, A./Jansen, B. J./Saracevic, T.: Vox populi: The public searching of the web. In: Journal of the American Society of Information Science, 52 (12), 2001, pp. 1073-1074. 